\title{
STRUCTURE OF ECAP ALUMINIUM AFTER DIFFERENT NUMBER OF PASSES
}

\author{
LuCia Ilucová ${ }^{1}$, IVAn SAXL ${ }^{1}$, Milan SvOBOdA ${ }^{2}$, VÁClav SKLENIČKA ${ }^{2}$ AND PeTr KRÁL ${ }^{2}$ \\ ${ }^{1}$ Mathematical Institute, Academy of Sciences of the Czech Republic, Žitná 25, CZ-115 67, Praha and Faculty of \\ Mathematics and Physics, Charles University, Sokolovská 83, CZ-18675, Praha, Czech Republic; ${ }^{2}$ Institute of \\ Physics of Materials, Academy of Sciences of the Czech Republic, Žižkova 22, CZ-616 62, Brno, Czech \\ Republic \\ e-mail:saxl@math.cas.cz; ilucova@math.cas.cz; svobm@ipm.cz; sklen@ipm.cz,pkral@ipm.cz \\ (Accepted February 6, 2007)
}

\begin{abstract}
The structure of high purity (99.99\%) aluminium processed by equal channel angular pressing in the as pressed state after different number of passes was examined using various stereological methods. An extreme inhomogeneity and complicated anisotropy was observed along the body of rod-like specimens.
\end{abstract}

Keywords: anisotropy, equal channel angular pressing (ECAP), non-homogeneity, orientation imaging microscopy (OIM).

\section{INTRODUCTION}

The interest in technological procedures severely refining the grain size of polycrystals arose due to the belief that they produce a considerable improvement of the mechanical properties of technical materials (Zhu et al., 2004). Equal channel angular pressing (ECAP) introducing large plastic strains into a coarse-grained material, typically pure metal or an alloy, belongs to the most promising ones. The material is subjected to a repeated severe plastic strain without any concomitant change in the cross-sectional dimensions of the work piece and this way a very significant refinement of polycrystalline grain size is attained.

In the preceding studies Ilucová et al. (2004) and Sklenička et al. (2006) was examined the structure of pure aluminium $(99.99 \%$ ) after a particular pressing process (consisting in eight passes of a rod-shape billet through the channel bent at a right angle and then, before the next pass, rotated by $90^{\circ}$ in the same direction - so-called route $\mathrm{B}_{\mathrm{C}}$ ) and then also after the subsequent creep deformation (performed at $473 \mathrm{~K}$ after an annealing for $4 \mathrm{hrs}$ under no external load before creep testing) (Sklenička et al., 2005; 2006; Saxl et al., 2007).

The present study is devoted to a better understanding of the effect of different number $N$ of repeated passes on the grain structure in the as pressed state (the same route $\mathrm{B}_{\mathrm{C}}$ was chosen). A considerable attention was also devoted to the examination of the subgrain and grain anisotropy and three mutually perpendicular plane sections were examined. However, it was already shown in Beneš and Saxl (2005) that three such planes are not sufficient for the description of spatial (3D) anisotropy. That is why only the profile (2D) anisotropy was determined by means of the intercept count along several directions in every section plane. The orientation of the profile boundaries was then described by the rose of directions and, equivalently, by the corresponding Steiner compact (Beneš and Saxl, 2005; Rataj and Saxl, 1989).

\section{EXPERIMENTAL RESULTS}

\section{SPECIMEN PREPARATION, ORIENTATION AND EXAMINATION OF PLANAR SECTIONS}

The repeated pressing of high purity (99.99\%) aluminium was conducted at room temperature and the metallographic examination was carried out by means of scanning (Philips SEM 505) electron microscope. Two samples were selected in each specimen and they are denoted by $\mathrm{B} N \_i, N=2,4,8,12$ and $i=1,2$. The Cartesian coordinate axes have been chosen with respect to the pressing procedure, namely $X$ along the last pressing direction and $\mathrm{Z}$ perpendicular to the bottom of the channel. Then three planar sections, namely $X Y, X Z$ (the longitudinal planes) and $Y Z$ (the transversal plane), selected at two different places were 
observed by means of the electron backscatter diffraction microscopy (EBSD or also OIM - orientation imaging microscopy) - Humphreys (2001). The images of planar sections were examined semi-manually by standard metallo-graphic methods, namely by profile as well as intercept counts supplemented by individual profile area estimation at four different disclinations (misorientation) $\Delta \geq 2^{\circ}, 5^{\circ}, 10^{\circ}$ and $15^{\circ}$. This approach made possible a detailed understanding of the subgrain and grain structure formation after various numbers of passes. In each of them were estimated the areas of individual profiles and the profile intensities $N_{A}(\bullet)$ (the mean number of profiles per unit area of the section plane; • stands for $X Y, X Z, Y Z$ ). The examples of sections are shown in Fig. 1. The values of chord intensities $N_{L}(\bullet)$ in the section planes (the mean number of chords per unit length of the test line) were determined as the arithmetic means of intercept counts in systematically selected six directions $\alpha_{k}=k \pi / 6, k=$ $0,1, \ldots, 5$, including the principal directions at $k=0,3$ in each section plane. As the samples $\mathrm{B} N \_1, \mathrm{~B} N \_2$ correspond to two different places of the same specimen selected at a distance of few millimetres only, their differences reflect the "large" scale inhomogeneity of specimens after $N$ passes. The total numbers of measured profiles in all sections were about 9000 , 17000,25000 and 14600 at $\Delta \geq 2^{\circ}$ and 600, 3500, 12000 and 6900 at $\Delta \geq 15^{\circ}$ in specimens B2, B4, B8 and $\mathrm{B} 12$, respectively.

The estimate of the grain intensity $N_{V}$ (the mean number of grains per unit volume) was obtained following the recommendation of the ASTM Standard (ASTM 1982, 1988) for anisotropic grain structures as $\left[N_{V}\right]_{\Pi N(A)}=0.81\left(\Pi N_{A}\right)^{3 / 2}$, where $\Pi$ denotes the geometric mean of $N_{A}(\bullet)$ (for a discussion of this relation and the comparison of the estimate $\left[N_{V}\right]_{\Pi N(A)}$ with the usual isotropic estimate $\left[N_{V}\right]_{\mathbf{E} N(A)}=0.81\left(\mathbf{E} N_{A}\right)^{3 / 2}$ (see Saxl et al., 2005). The surface intensity $S_{V}$ (the mean grain or subgrain boundary area per unit volume) was estimated as $\left[S_{V}\right]_{\mathbf{E} N(L)}=2 \mathbf{E} N_{L}$. Further, the estimate of the length intensity of grain boundary junctions $L_{V}$ (the mean length of triple grain or subgrain junction per unit volume) was estimated as $\left[L_{V}\right]_{\mathbf{E} N(A)}=4 \mathbf{E} N_{A}$, where $\mathbf{E}$ denotes the arithmetic mean with respect to all examined planes or directions (the relation $\left[S_{V}\right]=$ $2 \mathbf{E} N_{L}$ is the standard stereological relation (Stoyan et al., 1995), the relation $\left[L_{V}\right]=4 \mathbf{E} N_{A}$ follows from the fact that the mean number of profile vertices in a random $2 \mathrm{D}$ tessellation is 6 , hence $2 \mathrm{E} N_{A}$ estimates the mean number $P_{A}$ of triple points per unit section area and the estimate $\left[L_{V}\right]=2 P_{A}$ is again the standard stereological relation). Finally, the coefficients of variation of profile areas $a$ were estimated separately in the three section planes ( $\mathrm{CV} a(\bullet))$ as well as in the sample formed by all examined profile areas $(\mathrm{CV} a)$. 
$\Delta \geq 2^{\circ}$
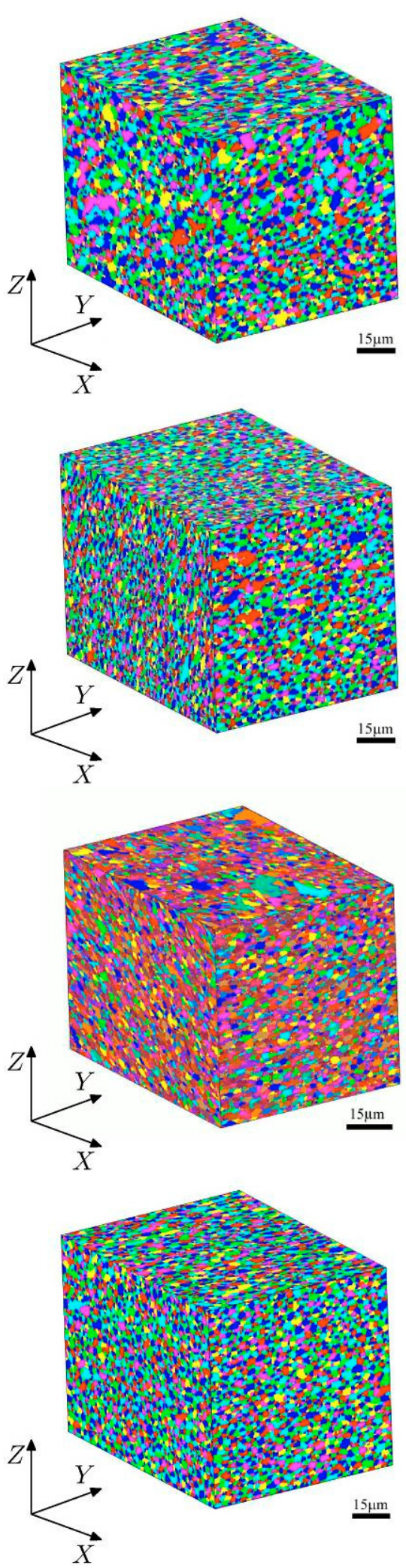

$\Delta \geq 10^{\circ}$
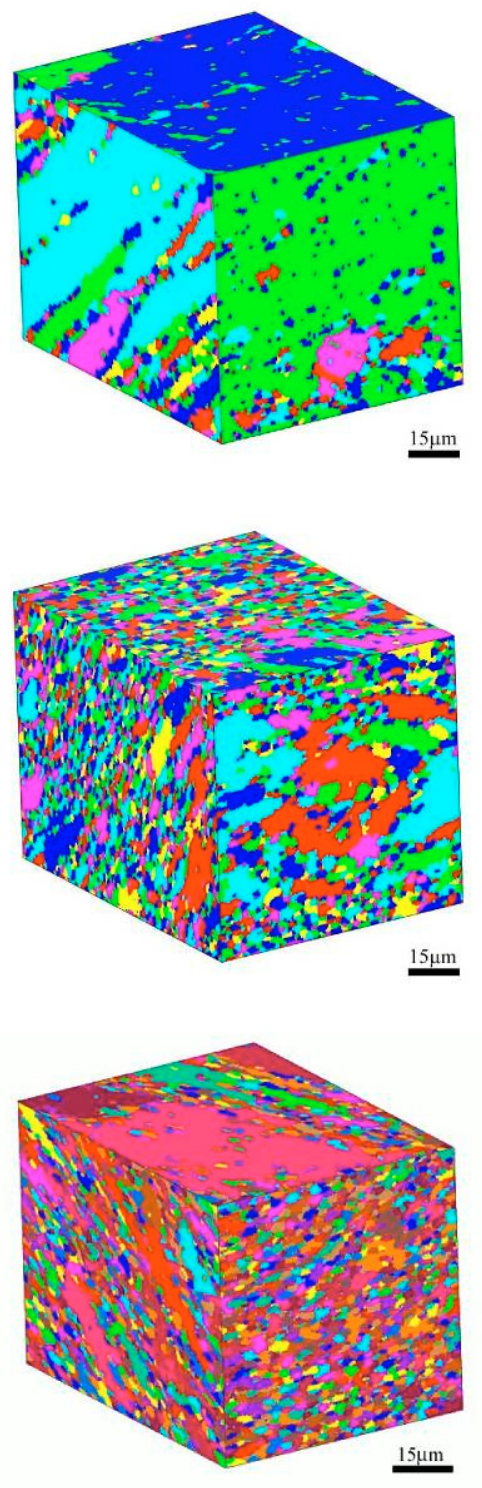

$15 \mu \mathrm{m}$

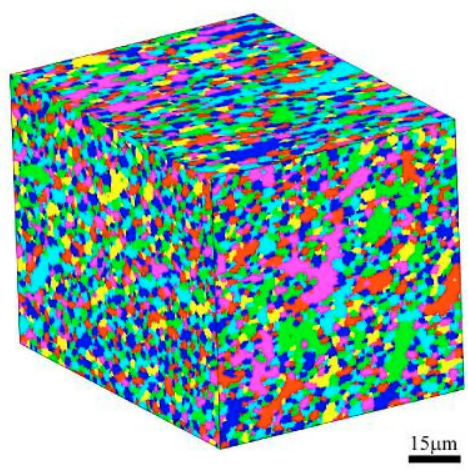

$\Delta \geq 15^{\circ}$
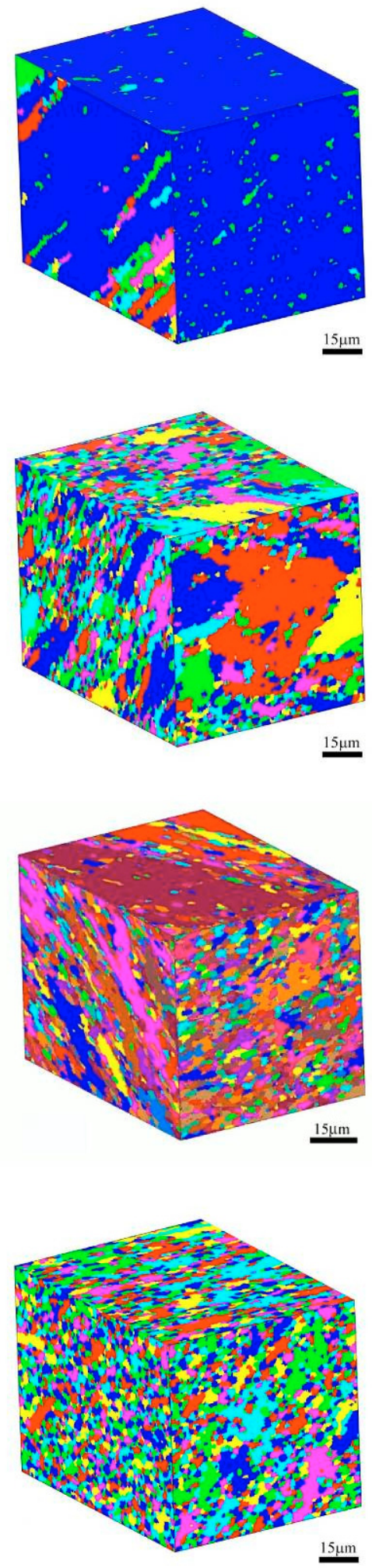

Fig. 1. Specimens B2_1 (1 $1^{\text {st }}$ row), B4_2 (2 ${ }^{\text {nd }}$ row $), B 8 \_1\left(3^{\text {rd }}\right.$ row) and B12_2 (last row) as observed at $\Delta \geq 2^{\circ}, \Delta \geq 10^{\circ}$ and $\Delta \geq 15^{\circ}$. 


\section{STEREOLOGICAL ESTIMATES OF 3D CHARACTERISTICS AND COEFFICIENTS OF VARIATION CV $A$}

The main obtained results are summarized in the Table 1; they include the estimates of 3D intensities and the profile area dispersions after different number of ECAP passes.

In general, the repeated pressing between $N=2$ and $N=12$ produces a considerable grain refinement of the order of $10^{2}$ (in the value of $N_{V}$ ). The total grain boundary area and the length of triple grain junctions increase accordingly. The local homogeneity as characterized by the values of $\mathrm{CV} a(\bullet)$ and $\mathrm{CV} a$ generally improves with the number of passes, but not systematically as clearly show the specimens B8_1 (the highest value of $\mathrm{CV} a$ ) and B8_2 (the lowest value of $\mathrm{CV} a$, perhaps an exceptionally homogeneous case see Fig. 2).

The ratios of the mean grain volumes $\mathbf{E} v\left(15^{\circ}\right) /$ $\mathbf{E v}\left(2^{\circ}\right)$ are about 70 and 12 in B2, B4 specimens, resp., and between 4 and 3 in the specimens after eight and twelve passes. Hence the mean number of subgrains included in a grain considerably decreases with the number of passes, which is also clearly visible by the visual inspection of section planes shown in Fig. 1. This reduction is caused mainly by the decreasing mean grain volume (roughly 100 times) and partly also by the increasing subgrain volume (roughly thrice) see below Table 3 .

Finally, the ratios of the mean CV $a$ estimated in two closely spaced places of the same specimen vary between 1 (B12) and 7 (B8). Consequently, a considerable homogenisation is achieved (perhaps?) only after 12 passes.

Also the estimates of $\left[N_{V}\right]_{\Pi N(A)}$ and $\left[N_{V}\right]_{\mathbf{E N}(A)}$ were mutually compared with the following result: the estimate based on the arithmetic mean is always higher but only in one case exceeds $150 \%$ of the $\left[N_{V}\right]_{\Pi N(A)}$ value. The reason of this relatively small difference is that the subgrain and grain anisotropies are not too pronounced.

Table 1. 3D estimates of the main grain characteristics $\left(\Delta \geq 15^{\circ}\right)$ and coefficients of variation CV a (*only one profile per observation window in the XZ plane, hence only the estimate of $\left[N_{V}\right]_{E N(A)}$ from $X Y$ and $Y Z$ sections is possible and the estimate $C V a(x z)$ cannot be calculated).

\begin{tabular}{lccccccc}
\hline & $\begin{array}{c}{\left[N_{V}\right]_{\Pi N(A)}} \\
{\left[\mu \mathrm{m}^{-3}\right]}\end{array}$ & $\begin{array}{c}{\left[S_{V}\right]_{\mathbf{E N ( L )}}} \\
{\left[\mu \mathrm{m}^{-1}\right]}\end{array}$ & $\begin{array}{c}{\left[L_{V}\right]_{\mathbf{E N ( A )}}} \\
{\left[\mu \mathrm{m}^{-2}\right]}\end{array}$ & $\mathrm{CV} a(x y)$ & $\mathrm{CV} a(x z)$ & $\mathrm{CV} a(y z)$ & CV $a$ \\
\hline B2_1 & 0.00240 & 0.12 & 0.10 & 8.01 & 6.44 & 14.24 & 10.00 \\
B2_2 & $0.00091^{*}$ & 0.03 & 0.04 & 6.87 & - & 10.68 & 8.10 \\
B4_1 & 0.01380 & 0.50 & 0.32 & 4.48 & 6.37 & 4.14 & 7.00 \\
B4_2 & 0.03900 & 0.69 & 0.48 & 3.17 & 2.65 & 6.62 & 4.80 \\
B8_1 & 0.08600 & 0.96 & 1.16 & 11.8 & 4.48 & 3.11 & 10.40 \\
B8_2 & 0.26000 & 1.69 & 2.01 & 1.51 & 2.45 & 1.41 & 1.50 \\
B12_1 & 0.07940 & 1.09 & 0.94 & 2.06 & 1.77 & 2.07 & 2.25 \\
B12_2 & 0.06110 & 1.00 & 0.74 & 2.30 & 1.31 & 2.78 & 2.31 \\
\hline
\end{tabular}
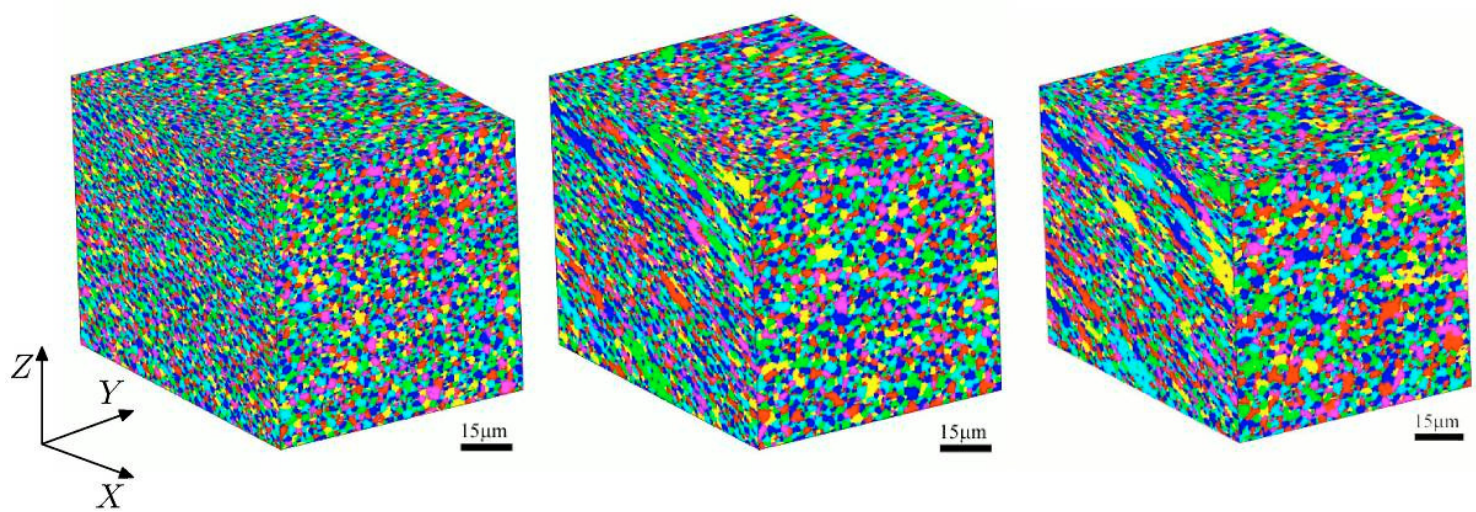

Fig. 2. The considerable homogeneity of grain size observed on the place $B 8 \_2$ at $\Delta \geq 2^{\circ}$ (left), $10^{\circ}$ (middle) and $15^{\circ}$ (right) even after 8 passes only. 
Whereas the $\left[N_{V}\right]_{\Pi N(A)}$ values are only rough estimates of the accurate $3 \mathrm{D}$ grain intensities, the estimates of the surface intensities $S_{V}$ and of the length intensities $L_{V}$ can be considered as rather accurate. They are presented in Table 1 for grains $\left(\Delta \geq 15^{\circ}\right)$. Finally, it must be stressed that the samples $\mathrm{B} N_{-} i, i=$ 1,2 at fixed $N=2,4,8$ are very different even when they are selected at closely spaced places of the same specimens. Thus the ratios of the $N_{V}$ estimates are roughly about $1: 3$ at a given $N=2,4,8$ and 1:1.3 at $N$ $=12$. Similarly the ratios of $S_{V}$ lie between 1:1.4 and $1: 1.1$ whereas the ratios of $L_{V}$ vary between 1:2.5 and $1: 1.3$; the lower bounds correspond to the B12 specimen.

In Table 2, the values of $S_{V}, L_{V}$ are the fractions of the values at $\Delta \geq 2^{\circ}$ corresponding to three ranges of disclination: subgrains with mutual disorientation between $2^{\circ}$ and $10^{\circ}$, intermediate range $\left[10^{\circ}, 15^{\circ}\right)$ and grains for $\Delta \geq 15^{\circ}$.

Combining the values from the both tables, the estimates of $\left[S_{V}\right]_{\mathbf{E} N(L)}$ and $\left[L_{V}\right]_{\mathbf{E} N(A)}$ for the examined disclination ranges can be simply calculated, e.g., $\left[S_{V}\right]_{\mathbf{E}(L)}=1.33 \mu \mathrm{m}^{-1}$ for the disclination range $2^{\circ} \leq \Delta$ and $0.25 \mu \mathrm{m}^{-1}$ for the disclination range $10^{\circ} \leq \Delta$ in the specimen B2_1. Moreover, the Table 2 reveals an important feature of ECAP structures after different number of passes, namely that the relative amount of high angle boundaries remarkably increases after each pass; they represent only about $10 \%$ and less at $N=2$ and exceeds $50 \%$ at $N=8$ and 12 . Equivalently, the inverse values of the estimated intensities $1 /\left[N_{V}\right]_{\Pi N(A)}$ $=[\mathrm{Ev}]$, namely the mean grain volumes at different disclination bounds can be compared - see Table 3 .

The mean size of all observed subgrains, i.e., with $\Delta \geq 2^{\circ}$, decreases only slowly with growing $N$, whereas a more substantial reduction of $[\mathbf{E} v]$ takes place at higher disclination values (approximately 4, 14 and 50 times at $\Delta \geq 5^{\circ}, 10^{\circ}$ and $15^{\circ}$, respectively). Consequently, the mean number of subgrains filling a grain is between 50 and 80 at $\Delta \geq 2^{\circ}$ and $8 \div 14,3 \div 4$ and 3 at $\Delta \geq 5^{\circ}, 10^{\circ}$ and $15^{\circ}$, respectively.

\section{ANISOTROPIC CHARACTERISTICS OF THE ECAP SPECIMENS}

The estimates of intensities $N_{L}(\alpha)$ obtained in six directions in each section plane can be considered as the support functions $u(\alpha+\pi / 2)$ (i.e., in the directions perpendicular to the test lines) of certain centrally symmetric convex polygon called the Steiner compact: the lengths of its edges are proportional to the relative weights of the directional measures of the examined fibre process. Hence the shape of the Steiner compact is in a certain way a mean shape of the profiles. When the estimate of the Steiner compact is made, the length of the edges are measured and used to construct the rose of direction of the profile boundaries. Roses of directions and Steiner compacts of profile boundaries observed in the chosen specimens $\mathrm{B} N i, N=2,4,8,12$ and $i=1,2$, at different lower bounds of disclination $\Delta$ are shown in Fig. 3.

Table 2. Fractions of grain and subgrain boundary areas and triple grain edge lengths.

\begin{tabular}{|c|c|c|c|c|c|c|c|c|}
\hline \multirow{2}{*}{ range of $\Delta$} & \multicolumn{8}{|c|}{ fractions of $S_{V}, L_{V}[\%]$} \\
\hline & B2_1 & B2 22 & B4_1 & B4_ 2 & B8_1 & B8_ 2 & B12_1 & B12_2 \\
\hline $2^{\circ} \leq \Delta<10^{\circ}$ & $81, \mathbf{8 5}$ & 93,93 & 52,67 & $44, \mathbf{6 3}$ & 32,40 & 24,43 & $24, \overline{40}$ & $29, \overline{42}$ \\
\hline $10^{\circ} \leq \Delta<15^{\circ}$ & 10,7 & 3,2 & $15, \mathbf{1 3}$ & 16,15 & 12,15 & 6,9 & $10, \mathbf{1 2}$ & $9, \mathbf{1 3}$ \\
\hline $15^{\circ} \leq \Delta$ & 9,8 & 4,5 & 33,20 & 40,22 & 56,45 & 70,48 & 66,48 & 62,45 \\
\hline
\end{tabular}

Table 3. The mean subgrain and grain volumes at different values of the disclination bounds.

\begin{tabular}{clrllllll}
\hline \multirow{2}{*}{ number of passes } & \multicolumn{1}{l}{$[\mathbf{E v}]\left[\mu \mathrm{m}^{3}\right]$} \\
\cline { 2 - 9 } & $\Delta \geq 2^{\circ}$ & \multicolumn{1}{c}{$\Delta \geq 5^{\circ}$} & \multicolumn{1}{c}{$\Delta \geq 10^{\circ}$} & \multicolumn{1}{c}{$\Delta \geq 15^{\circ}$} \\
\hline 2 & 7.46 & 12.40 & 26.30 & 200 & 147 & - & \multicolumn{1}{c}{417} & 1099 \\
4 & 5.00 & 3.14 & 13.20 & 6.72 & 31.15 & 13.96 & 72.50 & 25.60 \\
8 & 2.65 & 1.27 & 3.66 & 2.20 & 7.09 & 2.89 & 11.60 & 3.84 \\
12 & 3.96 & 4.80 & 6.00 & 7.20 & 8.78 & 11.20 & 12.60 & 16.40 \\
\hline
\end{tabular}


B2_1
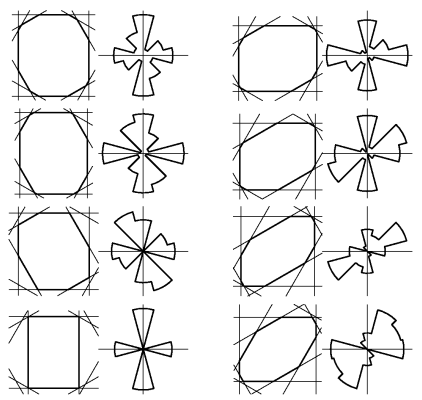

B8_1

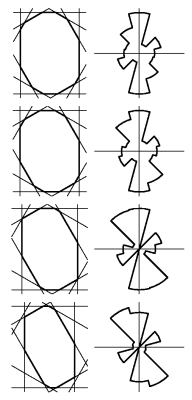

XY

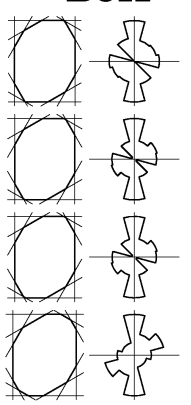

$\mathrm{XZ}$
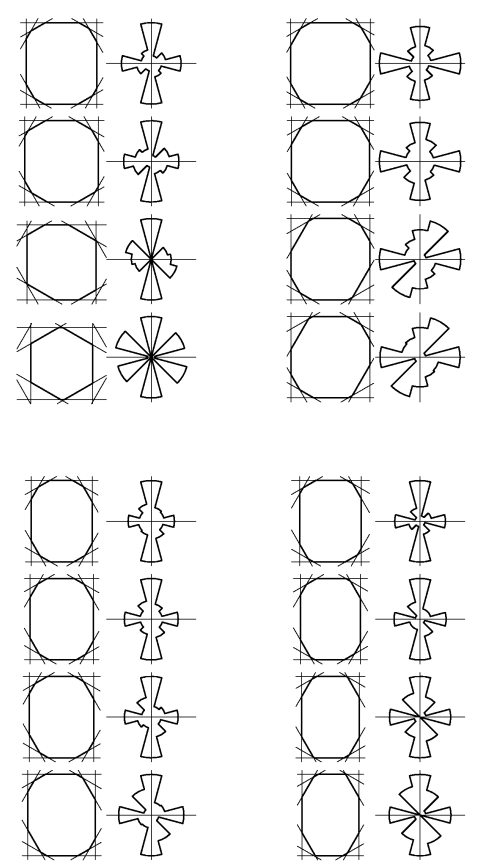

$\mathrm{YZ}$

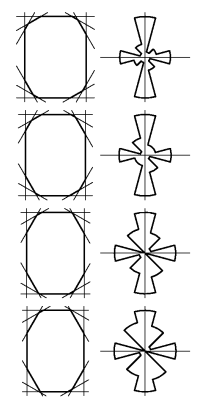

XY
B4_2

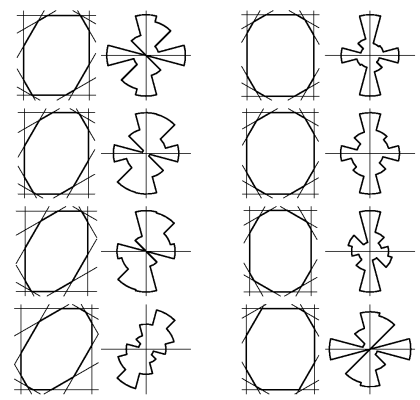

B12_2

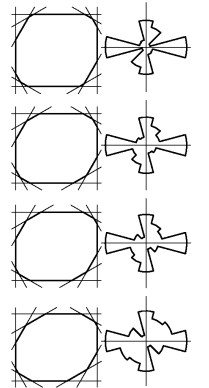

XZ

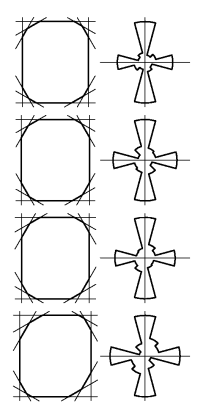

$\mathrm{YZ}$

Fig. 3. Steiner compacts and roses of directions of sub-boundary and boundary profiles of specimens B2, B4, $B 8$ and $B 12$ in the section planes $X Y, X Z$ and $Y Z$ at the disclinations $\Delta \geq 2^{\circ}$ (upper row), $\Delta \geq 5^{\circ}, \Delta \geq 10^{\circ}$ (middle rows), $\Delta \geq 15^{\circ}$ (lower row) - identically for all specimens. The Y-axis is always vertical, the Z-axis always horizontal.

The profiles boundaries approximately follow the chosen coordinate axes in the $X Y$ and $Y Z$ planes and the profile elongation along the transverse axis $Y$ is preponderant, which is somewhat surprising in the $X Y$ plane as $X$ is the pressing direction. On the other hand, profiles inclined with respect to the coordinate axes and elongated along the $X$ direction prevail in the $X Z$ plane. The subgrains as well as grains are approximately plate-like, usually inclined with respect to the $Z$-axis.

This graphical anisotropy characterization can also be supplemented by the eccentricities of the correspon- ding Steiner compacts that are defined as the ratio $\varepsilon=(\max w / \min w)-1$, where $w$ is the width of the Steiner compact (i.e., the distance of its support lines). The maximum (with respect to the both examined places) values in B2, B4 and B12 specimens and the values for B8 1 specimen of eccentricities $\varepsilon(\bullet)$ are presented in Table 2. Also the orientation of the Steiner compact with respect to the coordinate axes is important. The directions of the both widths max $w$ and $\min w$ approximately coincide with the coordinate axes; otherwise the value of the eccentricity in Table 4 is printed in bold letters.

Table 4. Eccentricities of Steiner compacts in the different section planes; usually the maximum and minimum widths of the compacts correspond to the coordinate axes $X, Y$ and $Z$. If the compact is severely inclined, the eccentricities are in the bold letters. The meaning of inequalities is that if, e.g., $Y>X$ then the width $w(Y)>w(X)$ even if the compact is inclined with respect to the coordinate axes.

\begin{tabular}{c|ccc|ccc|ccc|ccc}
\hline \multirow{3}{*}{$\Delta$} & \multicolumn{4}{|c|}{$\mathrm{B} 2$} & \multicolumn{4}{c|}{$\mathrm{B} 4$} & \multicolumn{3}{c|}{$\mathrm{B} 8 \_1$} & \multicolumn{3}{c}{$\mathrm{B} 12$} \\
\cline { 2 - 13 } & $\varepsilon(X Y)$ & $\varepsilon(X Z)$ & $\varepsilon(Y Z)$ & $\varepsilon(X Y)$ & $\varepsilon(X Z)$ & $\varepsilon(Y Z)$ & $\varepsilon(X Y)$ & $\varepsilon(X Z)$ & $\varepsilon(Y Z)$ & $\varepsilon(X Y)$ & $\varepsilon(X Z)$ & $\varepsilon(Y Z)$ \\
& $Y>X$ & $X>Z$ & $Y>Z$ & $Y>X$ & $*$ & $Y>Z$ & $Y>X$ & $X>Z$ & $Y>Z$ & $Y>X$ & $X>Z$ & $Y>Z$ \\
\hline $2^{\circ}$ & 0.22 & $\mathbf{0 . 4 2}$ & 0.24 & 0.20 & $0.43^{\dagger}$ & 0.38 & 0.35 & $\mathbf{0 . 4 8}$ & 0.53 & 0.33 & 0.30 & 0.34 \\
$5^{\circ}$ & 0.29 & $\mathbf{0 . 5 4}$ & 0.32 & 0.24 & $0.59^{\dagger}$ & 0.49 & $\mathbf{0 . 3 7}$ & $\mathbf{0 . 5 1}$ & 0.31 & 0.34 & 0.35 & 0.33 \\
$10^{\circ}$ & $\mathbf{0 . 3 8}$ & $\mathbf{0 . 9 7}$ & 0.20 & $\mathbf{0 . 1 9}$ & $1.15^{\dagger}$ & 0.53 & $\mathbf{0 . 4 4}$ & $\mathbf{0 . 4 3}$ & 0.31 & 0.41 & 0.38 & 0.29 \\
$15^{\circ}$ & $\mathbf{0 . 7 3}$ & $\mathbf{0 . 8 0}$ & 0.20 & $\mathbf{0 . 2 6}$ & $\mathbf{1 . 1 5}$ & 0.50 & $\mathbf{0 . 6 0}$ & $\mathbf{0 . 4 2}$ & $\mathbf{0 . 4 4}$ & 0.47 & 0.38 & 0.28 \\
\hline
\end{tabular}

* $X>Z$ in B4_1 and $Z>X$ in B4_2, ${ }^{\dagger}$ inclined only at $Z>X$ 
The greatest eccentricities and the more pronounced inclinations are observed in the $X Z$ plane, the most isotropic are profile boundaries in the $Y Z$ transverse planes. The anisotropy of subgrains as characterized by the profile eccentricity increases with the growing disclination in the $X Y$ and $X Z$ planes and is nearly constant in the $Y Z$ planes. The highest eccentricities are observed after two and four passes and the isotropy improves with the increasing number of passes.

\section{CONCLUSIONS}

The consequence of the increasing number of passes between $N=2$ and $N=12$ is the substantial grain refinement of pure ECAP aluminium products. It is simultaneously accompanied by an increasing fraction of the high angle grain boundaries. The local as well as overall homogeneity of ECAP products also improve considerably with the number of passes but even after 8 passes is not achieved. The difference between specimens after 8 and 12 passes consists mainly in a more pronounced homogeneity of the latter case.

The anisotropy of grain and subgrain boundaries is rather mild and also improves with the number of passes. The grain boundaries are slightly more anisotropic than their subgrains, plate-like grain shapes elongated in $X$ and $Y$ directions usually prevail but the plate normals are frequently inclined with respect to the coordinate axes.

\section{ACKNOWLEDGMENT}

The authors acknowledge the financial support for this work provided by the GAAV ČR (grant A 204 1301), by the GA ČR (grant 201/06/0302), by the grant MSM 0021620839 and by the Academy of Sciences of the Czech Republic, Institutional Research Plan No. AV0Z10190503.

This paper is an extended version of the contri- bution Inhomogeneity and anisotropy of ECAP Aluminium presented at the S4G International Conference on Stereology, Spatial Statistics and Stochastic Geometry, Prague, June 26-29, 2006.

\section{REFERENCES}

ASTM (1982, 1988). Standard methods for determining average grain size. ASTM E 112.

Beneš V, Saxl I (2005). Stereological estimation of the rose of directions from the rose of intersections. In: Baeza-Yates R, Glaz J, Gzyl H, Hüsler J, Palacios JL, eds. Recent Advances in Applied Probability. SpringerVerlag, 65-96.

Humphreys FW (2001). Grain and subgrain characterisation by electron backscatter diffraction. J Mat Sci 36:3833-54.

Ilucová L, Král P, Svoboda M, Saxl I, Sklenička V (2004). Estimation of grain size and size inhomogeneity in ultrafine-grained aluminium processed by ECAP method. In: Gundlach C et al., eds. Evolution of Deformation Microstructures in 3D. Proceedings of the 25th Risø International Symposium on Materials Science, 2004 Sep 6-10; Roskilde. Risø National Laboratory, 363-8.

Rataj J, Saxl I (1989). Analysis of planar anisotropy by means of the Steiner compact. J Appl Probab 26:490-502.

Saxl I, Ponížil P, Sülleiová K (2005). Grain size estimation in anisotropic materials. Mat Sci Forum 482:239-53.

Saxl I, Sklenicka V, Ilucova L, Svoboda M, Kral P (2007). Structure Development during ECAP and Subsequent Creep of Aluminium. Mat Sci Forum 493-498:493-8.

Sklenička V, Dvořák J, Král P, Stonawská Z, Svoboda M (2005). Creep processes in pure aluminium processed by equal-channel angular pressing. Mater Sci Eng A 410-411:408-12.

Sklenička V, Král P, Ilucová L, Saxl I, Dvořák J, Svoboda $M$ (2006). Inhomogeneity of microstructure and creep of ECAP Aluminium. Mat Sci Forum 503:245-50.

Stoyan D, Kendall WS, Mecke J (1995). Stochastic Geometry and its Applications. New York: J Wiley \& Sons.

Zhu YT, Langdon TG, Valiev RZ, Semiatin SL, Shin DH, Lowe TC, eds. (2004). Ultrafine Grained Materials III. Warrendale: The Minerals, Metals and Materials Society. 